\title{
Correction to: Antifungal activity and bean growth promotion of Trichoderma strains isolated from seed vs soil
}

\author{
S. Mayo-Prieto • M. P. Campelo • A. Lorenzana • \\ A. Rodríguez-González • B. Reinoso • S. Gutiérrez • \\ P. A. Casquero
}

Published online: 3 December 2020

(C) Koninklijke Nederlandse Planteziektenkundige Vereniging 2020

\section{Correction to: Eur J Plant Pathol https://doi.org/10.1007/s10658-020-02069-8}

Article PDF was incorrectly paginated in Volume 158, Issue No.4.

First page should read as 817 and last page 828 .

Original article has been updated.

The online version of the original article can be found at https://doi.org/10.1007/s10658-020-02069-8

S. Mayo-Prieto $(\varangle) \cdot$ M. P. Campelo • A. Lorenzana •

A. Rodríguez-González • B. Reinoso • P. A. Casquero Grupo Universitario de Investigación en Ingeniería y Agricultura Sostenible (GUIIAS), Instituto de Medio Ambiente, Recursos Naturales y Biodiversidad, Universidad de León, Avenida Portugal 41, 24071 León, Spain

e-mail: smayp@usnileon.es

S. Gutiérrez

Grupo Universitario de Investigación en Ingeniería y Agricultura Sostenible (GUIIAS), Área de Microbiología, Escuela de Ingeniería Agraria y Forestal, Universidad de León, Campus de Ponferrada, Avenida Astorga s/n, 24400 Ponferrada, Spain 